\title{
SOCIOMETRY AND FAMILIAL STATUS-ROLE RELATIONS
}

\begin{abstract}
Being one of the contemporary social psychology directions, sociometry has played a vital role in small group investigations. That direction was formed, developed and widely applied in the first decades of the 20th century by Moreno who was first of all concerned with primary, i. e. intimate, emotional relations of people in small groups, emergence of feelings among group members such as sympathy and dislike, love and hatred, respect and contempt, the desire to be in mutual communication or isolation from each other, etc. With the help of the sociometric method the latter tries to reveal such inter-group relations which are hardly perceptible for "naked eyes" (to observe superficially), however they often have serious, sometimes also critical influence on people's behavior and state of mind. And though there has been put forth the viewpoint that sociometry is not as efficient in family investigation as it is with other small and medium group studies, nevertheless, family sociometric research is compulsory and useful for investigating family psychology.
\end{abstract}

Keywords: sociometric statuses, family, relations, social group, psychodrama, cognitive roles, sociogramm.

The status-role approach to family issues would not be complete if we were content with the investigation of only that social group: "official" statuse and roles reveal their mutual relations and role socialization processes. Social psychologists, primarily Jacob Moreno, have shown that in each social group within the course of time emerge pure psychological, the so-called sociometric statuses and appropriate roles as a consequence of its members' interactions and the whole group dynamics. Despite of the the viewpoint that sociometry is not as efficient in family investigation as in case of studying other small and medium groups, family sociometric research is still compulsory and useful for investigating family psychology.

Being one of the contemporary social psychological directions, sociometry has played a vital role in small group investigations. That direction was formed, promoted and widely applied in the first decades of the 20th century by Moreno who was first of all concerned with primary, i. e. intimate, emotional relations of people in small groups, emergence of feelings among group members such as sympathy and antipathy, love and hatred, respect and contempt, the desire to be in mutual communication or isolation from each other, etc. With the help of the sociometric method the latter tries to reveal such inter-group relations that are hardly perceptible to the "naked eye" (to observe superficially), however they often have serious, sometimes also critical influence on people's behavior and state of mind. (Volkov 1970; Moreno 1958).

Unlike other representatives of role theory (Mead and others), Moreno shaped his notions about social statuse and roles as a result of lasting and rich practical work. He implemented this experimental investigations using the method of psychodrama created by himself. Being a psychiatrist, Moreno was first of all more concerned with active people, their activities and interrelations, and lesswith society structure, official roles and sanctions. In his works practical issues and theoretical deductions are interlinked. Moreno studies also specific social role performers who act in specific situations 
("in situ" as Moreno likes to say). In order to give a name to these relations he promoted the concept of "sociometric networks". In small groups he studied people's interrelations as the main subject for his interests. Still in 1914 he suggested the idea of accepting another person's role while people communicate. By saying "role" he meant spontaneous and emotional activeness of an individual with a person's in-depth involvement in that activity. It is necessary to mention that in G. Meads' works role and the process of accepting it are mostly perceived as intellectual processes. He considers language and speech as a result of role performance, too, and not preconditions of role performance as we see in Meads' theory. (Moreno 1968. ).

According to Moreno the concept of "role" is filled with psychological content, while some sociologists oppose this idea, hence, in their opinion the investigation of the concept "role" should be taken up by sociologists rather than by psychologists. Emphasizing spontaneous and creative characteristics of social roles Moreno contradicts Mead's theory stating that a person is always in conflict with his own official roles. One of the best ways of getting rid of such a conflict is to assume unofficial (spontaneous, sociometric) roles. It is in the process of realization of these roles that a person gets the opportunity to fully demonstrate his personality. An individual creates his roles himself and while performing them creatively he solves his problems.

Moreno made those ideas as the basis of one of his best methods in psychotherapypsychodrama, a method that is also expedient to use for handling inter-familial problems. In 1934, Moreno properly described the processes of role, its acceptance and implementation in his work entitled , "Who experiences emotions?" where he has already promoted the outlook that while performing spontaneous roles, people can also change society structure.

Moreno has not made detailed characterization of social roles. He has not linked people's roles with formal rights and duties either. Those issues were dealt with by other social investigators such as Nucom, Linton and others. Moreno was particularly interested in specific processes of role performance in certain place and period. However we can state that in his works there is a complete miss of the comprehension and social functions that can be ascribed to the concept "role". In Moreno's works interesting ideas about relations between roles and a person's self-consciousness exist. In addition, he also comes to the conclusion that role performance is the particular demonstration of a person and his own self. Some social psychologists think that the above-mentioned ideas of Moreno allow us to pass from dramatic level of the role to its social level. However Moreno did not take into account the circumstance that spontaneous role performance can also be a realization process of attributed and role expected by referential groups.

Although both social psychologists and family specialists think that sociometry cannot be that efficient in family investigation as in small and medium group studies, it provides very important data about more delicate and hidden, somehow not realized inter-familial relations. For revealing those intrapersonal and emotionally saturated relations and positions they use a special method-sociometric test that allows one to discover the unofficial sociometric status of each group member. Each individual's sociometric status indicates what role he plays in the network of unofficial group relations. Moreno has called those statuses (from the highest to the lowest one) star, preferred, accepted, isolated and rejected. Those statuses are determined according to the ratio of positive and negative selections of each person. As far as sociometric methodology is concerned, the ways of its application have been widely illustrated in the relevant literature, it is not considered necessary to introduce them here. It is noteworthy that each individual performs roles appropriate for sociometric status in a group and in a family. Without a special investigation we can already state that one of the family members (e.g. the youngest child) can be the favor- 
ite of each member (this is the position of star), and the other may just be accepted and give birth to warm feelings among others, etc.

In the field of family psychology the application of sociometry seems promising to us, particularly for studying extended families still existing in rural areas.

Unfortunately as far as we are informed this direction is not developing. However in the works of Armenian psychologists there have been enquiries about the necessity of its development. In different countries, especially in Russia and Belorussia there have been conducted wide-scope researches in schools. Those researches assist one to reconstruct human interrelations, change some people's unfavorable positions and roles causing mutilation. They sometimes have positive psychiatric impact on individuals and groups as well.

There are a lot of new issues that can be solved in the field of family psychology due to sociometric investigations. One of them is to find the types of interactions that are among family members' official and unofficial (sociometric) status and relevant roles and the impact of those interactions on observable behavior. Moreover, those interactions and their consequences must be studied at the level of both distinct people and the whole group. The following can be as an assumption: there are two kinds of interactions of the above mentioned types, positions and roles (if we don't observe possible neutral state): a) mutual reinforcement (positive interaction) and b) mutual inhabitation (negative interaction). Study shows that for the investigation of those interrelations an appropriate methodology is necessary to work out.

The next thesis (and issue) is that in our opinion a person's role performance in a family cannot be only considered as an expression of his assumed official status. Sociometric status impacts on role behavior mostly at subconscious level, but there can always be perceived the traces of those impacts on behavior. For instance, pedagogues require parents to treat all children equally. A parent can agree with this and try to treat in that way, but his sociometric position interacting with each child's sociometric position at the subconscious level spontaneously results in behavior differences. This is one of the phenomena that is usually called "subjectivism".

The application of sociometric classic methodology in the family investigation is connected with serious difficulties as there are few modern nuclear families and the existence of all the sociometric statuses is not possible. Besides, members of a small family, especially spouses have already chosen each other, and asking them questions like "Who would you like to marry if you had a free choice" would be inappropriate and irrelevant. Taking into consideration this and many other situations psychotherapists have created a special family sociogram (similar to Moreno's classic sociogram) which is being introduced to each family member before psychotherapy and in different stages of that process (to the "problematic person" of a family). (Eidemiller, Dobryakov, Nikolskaya 2003).

The methodology enables us to somehow reveal sociometric methodology which remains not realized. Both the above-mentioned and other authors claim that this method is quite efficient and essentially useful for psychotherapists. The subjects (people who are studied) are given forms in each of which there are drawn circles with 110 diameters. The subject is instructed to place both himself and the other members of his family in the shape of circles with different diameters inside the circle. Each subject is assigned to do the work independently without consulting with others. It covers particular sides of familial life before psychotherapy and the kind of data that can be received from sociogram, and the spheres a psychologist should pay attention to? It turns out that it can provide useful information about the following:

1. About the family members presented in the circle,

2. The sizes of circles showing family members,

3. Arrangement of circles towards each other, 
4. Distance of circles from each other.

It turns out that those signs and their reciprocal relations are quite informative from psychological point of view. After completing the work the subject must sign under the sociogram.

The number of circles: an investigator must first of all pay attention to the number of persons family consists of and how many people are introduced in the sociogram. However surprising it might seem there are subjects that don't present all their family members in the circle. It implies that not all his family members are significant for him or some are rejected. Such "oblivion" can also imply conflicting relations between the subject and the person in oblivion. Moreover, some subjects include people in circles who are not members of that family. Sometimes they include even their pets.

The size of circles: The main principle is that the bigger the circle introducing a person, the more important and significant he is considered. For example, if a subject presents himself in a bigger circle compared to others it speaks about his very high self-esteem. Otherwise we can conclude that he underestimates himself. Let's mention that interpretation of this peculiarity produces truer results when the subject doesn't know for what purpose the investigation is conducted. Only in this case he will freely demonstrate the psychological information that is interesting for a psychologist and psychotherapist.

The arrangement of circles: In the case of each individual that arrangement is certainly different. If he places a family member in the corner of the circle (testing field) and in a very small size it implies that he isolates or rejects him. Subjects present important members of their family (from the point of view of an individual ) in the center of the testing field and in bigger size. From psychological perspective it's important where the subject places himself and in which size. If he places his "self" in the center of a big circle and in big sizes, it can be concluded that he has well-expressed egoism and high selfesteem. And if he presents himself in the corner and in small sizes he is considered to be oppressed and to have a low self-esteem.

The distance between circles: That index is related to emotion interactions among family members, subconscious peculiarities of the subject. The main principle of interpretation is that the closer some members of a family are placed, the closer they are emotionally. If he places himself and a member of his family so closely, he considers that member as a close person. And vice versa, large distance between himself and another person in the circle symbolizes alienation between them. If in a circle family members are placed far from each other it can be assumed that there are conflicting relations between them, reciprocal emotional rejecting, little or lack of desire to communicate with each other.

That peculiarity can imply some level of positive psychological identity between a subject and another person. If, e.g. a subject places himself and his mother in the form of intersecting circles, some authors (Eidemiller, Olifirovich and others) interpret it as evidence that he hasn't fully distinguished his "self" from his mother 's self. And this is a sign of insufficient level of development and maturity of a person. Such a tie between two people is called "symbiotic", however it is mostly related to psychological unity, lack of distinctiveness. As far as a family is a small, but dynamic system, as it can be expected, relationships undergo changes there with time. They can be observed during psychotherapy, too. Those changes are somehow reflected in sociogram. Sociograms presented by the same person also differ in different stages of family life, particularly in conditions of normative and other types of conflicts. 


\section{REFERENCES}

Eidemiller, E., Dobryakov, I., Nikolskaya. I. (2003). Family diagnosis and family psychotherapy. Handbook for doctors and psychologists. St. Pet.

Moreno, J. (1968). Sociometry. Moscow: Politizdat.
Sedrakyan, S. (2014).Family Psychology. USA,

Volkov, I. (1970). Sociometric methods in socialpsychological investigations. Leningrad, Ed. In MSU.

Moreno, J. (1958). Sociometry. Moscow: "Politizdat". 